\title{
Die Projektion der langfristigen Entwicklung der gesetzlichen Rentenversicherung
}

\section{Methodische Vorgehensweise und empirische Ergebnisse}

\author{
Valentin Vogt
}

\section{Zusammenfassung}

Der demografische Wandel stellt die Nachhaltigkeit der umlagefinanzierten sozialen Sicherungssysteme in Deutschland grundsätzlich in Frage. Unter Nachhaltigkeit der sozialen Sicherung ist dabei die Gewährleistung eines bestimmten Sicherungsniveaus bei gleichzeitiger Stabilisierung des Beitragssatzes und des Bundeszuschusses zu verstehen. Durch eine anhaltend niedrige Geburtenrate und einen starken Anstieg der Lebenserwartung verändert sich das Verhältnis zwischen Beitragszahlern und Leistungsempfängern zu Lasten der Erwerbstätigen. In diesem Beitrag wird ein Simulationsmodell zur langfristigen Analyse der Nachhaltigkeit der gesetzlichen Rentenversicherung (GRV) in Deutschland vorgestellt. Es besteht aus einer Bevölkerungsprojektion, einem makroökonomischen Wachstumsmodell und einer detaillierten Berechnung der Einnahmen und Ausgaben der gesetzlichen Rentenversicherung. Die Projektionen zeigen, dass der Beitragssatz zur GRV bereits in den kommenden Jahren deutlich ansteigen wird, und eine langfristige Stabilisierung der gesetzlichen Rentenversicherung ohne größere Reformen nicht möglich ist.

\section{$1 \quad$ Nachhaltigkeitsprobleme sozialer Sicherungssysteme}

Die Frage nach der Nachhaltigkeit sozialer Sicherungssysteme beschäftigt die Sozialpolitik bereits seit mehr als einem Jahrzehnt. So legte die Kommission „Nachhaltigkeit in der Finanzierung der sozialen Sicherungssysteme“ im Jahr 2003 ihren Bericht vor, der erhebliche Einschnitte im Sozialversicherungsrecht zur Folge hatte. 
Kurz darauf wurde der „Nachhaltigkeitsfaktor“ in die Rentenformel eingefügt und die bisherige "Schwankungsreserve" in "Nachhaltigkeitsrücklage“ umbenannt.

Der Grund für die intensive Nachhaltigkeitsdiskussion im System sozialer Sicherung ist die zu erwartende demografische Entwicklung in Deutschland, die die finanzielle Tragfähigkeit dieses Systems zu überfordern droht. Der Begriff der Nachhaltigkeit wird in diesem Zusammenhang also als finanzielle Stabilität der sozialen Sicherungssysteme gegenüber exogenen Einflüssen durch die demografische oder wirtschaftliche Entwicklung verstanden. Der Begriff der Tragfähigkeit beinhaltet dabei auch die Frage nach der Belastbarkeit privater Wirtschaftssubjekte mit Beiträgen zum System sozialer Sicherung, wobei ein ausreichendes Sicherungsniveau als Nebenbedingung zu berücksichtigen ist. Inwieweit die jüngsten sozialpolitischen Maßnahmen und auch die aktuelle Entwicklung im Bereich der Migration dazu beitragen, diese finanzielle Nachhaltigkeit der umlagefinanzierten sozialen Sicherungssysteme sicher zu stellen, kann jedoch nur im Rahmen umfangreicher, auf lange Fristen angelegter Simulationsrechnungen überprüft werden.

In diesem Beitrag sollen der grundlegende Aufbau und die wesentlichen Ergebnisse einer langfristigen Simulation der Auswirkungen der Bevölkerungsentwicklung auf die gesetzliche Rentenversicherung dargestellt werden. Sie ist als die größte der verschiedenen Sozialversicherungen von besonderem Interesse. Der Beitrag ist wie folgt gegliedert: Zunächst wird kurz der grundsätzliche Aufbau des Simulationsmodells beschrieben. Anschließend folgt eine etwas ausführlichere Beschreibung der Projektion der Bevölkerungsentwicklung und ihrer wesentlichen Komponenten. Der nächste Abschnitt stellt den Aufbau der ökonomischen Simulationsrechnungen des Modells dar. Schließlich wird ausführlich die Methodik der Budgetrechnungen der gesetzlichen Rentenversicherung beschrieben. Anschließend werden die Ergebnisse der Simulationen vorgestellt, wobei ein besonderes Augenmerk auf die Beitragssatzentwicklung gelegt wird.

\section{Modellaufbau}

Für die langfristige Finanzierbarkeit umlagefinanzierter sozialer Sicherungssysteme ist nicht nur der Einfluss des demografischen Wandels auf die Bevölkerungsgröße, sondern insbesondere auch seine Auswirkung auf die Altersstruktur der Bevölkerung relevant. Für beide Veränderungen sind im Wesentlichen drei Faktoren ausschlaggebend: die Lebenserwartung, das Geburtenverhalten und die Außenwanderungen. Werden sie adäquat fortgeschrieben, so lässt sich mit Hilfe einer auf der Technik der Leslie-Matrizen (Caswell, 2001: 8-31) basierenden Bevölkerungsprojektion eine 
relativ zuverlässige Prognose für einen Zeitraum von ca. 20 Jahren durchführen. Die hohe Prognosequalität über diese Zeitspanne liegt darin begründet, dass sich das Geburtenverhalten in der Vergangenheit meist nur recht langsam verändert hat, und dass Methoden zur Fortschreibung der Lebenserwartung für einen solchen Zeitraum noch eine recht gute Prognosequalität besitzen. Auch haben die für Fragestellungen der Rentenversicherung in diesem Zeitraum relevanten Geburten bereits zu einem großen Teil stattgefunden. Für einen längeren Zeitraum von etwa 50 Jahren sinkt die Eintrittswahrscheinlichkeit prognostizierter Entwicklungen jedoch deutlich ab. Dies gilt allerdings nur, falls sich beim Geburtenverhalten oder bei der Entwicklung der Lebenserwartung grundlegende Änderungen gegenüber bisherigen Trends vollziehen. Für Unsicherheit sorgt allerdings die geringe Prognostizierbarkeit der zukünftigen Migrationsströme aus dem und ins Ausland. Hier ist es nur möglich, im Rahmen von Szenarioanalysen die Bandbreite möglicher Ereignisse abzudecken.

Wesentlich weniger vorhersehbar als die demografischen Veränderungen ist die wirtschaftliche Entwicklung. Dazu kann eine ganze Reihe unterschiedlicher Komplexitätsstufen der Modellierung in Betracht gezogen werden. Die einfachste Methode besteht darin, das Arbeitsangebotsverhalten für alle Bevölkerungsgruppen als konstant zu unterstellen oder mit einem Trend aus der Vergangenheit fortzuschreiben. Die Entwicklung der Erwerbspersonenzahl folgt somit aus der prognostizierten Entwicklung der Bevölkerung und wird als einzige endogene Einflussgröße eines einfachen Wachstumsmodells betrachtet. Der technische Fortschritt, die Arbeitslosenquote sowie sämtliche weitere Einflussgrößen auf Wirtschafts- und Lohnwachstum werden dann entweder exogen fortgeschrieben oder mit einem als plausibel eingestuften Verlauf vorgegeben. Eine Endogenisierung der Auswirkungen der Anpassung der sozialen Sicherungssysteme an die demografischen und wirtschaftlichen Gegebenheiten auf die wirtschaftliche Entwicklung wäre dagegen nur im Rahmen eines allgemeinen Gleichgewichtsmodells möglich (Werding, 2011: 3). ${ }^{1}$

Das Simulationsmodell ist grundsätzlich in mehrere Module aufgeteilt, und beinhaltet die Möglichkeit, weitere Module wie beispielsweise die Modellierung weiterer Sozialversicherungsträger oder die Mikrosimulation eines Teilbereiches zu berücksichtigen. Die Basis für sämtliche Simulationsrechnungen bildet dabei das Bevölkerungsprojektionsmodul. Auf dieses baut das Modul „Erwerbspartizipation“

1 Ein solches allgemeines Gleichgewichtsmodell verwenden beispielsweise Auerbach \& Kotlikoff (1987) oder Fehr et al. (2005), allerdings auf der Basis von overlapping generations (OLG) anstatt einer vollständigen Bevölkerungsprognose. Diese Vorgehensweise eignet sich eher für theoretische Fragestellungen als für konkrete Budgethochrechnungen. 
auf. Die Ergebnisse beider Teile dienen anschließend dem Modul „Makroökonomisches Hintergrundszenario" als Grundlage. Auf alle drei Module baut dann schließlich die Simulation der gesetzlichen Rentenversicherung auf.

\subsection{Das Modul zur Bevölkerungsprojektion}

Das Bevölkerungsprojektionsmodul fußt auf der Technik der Leslie-Matrizen (hierzu Caswell, 2001: 8-31). Diese schreibt die nach Geschlecht und Alter unterteilte Bevölkerung eines bestimmten Basisjahres ${ }^{2}$ anhand der Eintrittswahrscheinlichkeit zweier zentraler Lebensereignisse fort. Dabei handelt es sich zum einen um die Überlebenswahrscheinlichkeit, und zum anderen um die Fertilität bzw. Geburtenwahrscheinlichkeit. Die Überlebenswahrscheinlichkeit ist dabei definiert als die Wahrscheinlichkeit, am Stichtag des Folgejahres noch am Leben und ein Jahr älter zu sein. Die Fertilitäten sind definiert als die Wahrscheinlichkeit, dass sich pro Frau einer Altersklasse zum Stichtag des Folgejahres ein zusätzliches Kind in der Altersklasse „Neugeborene“ des jeweiligen Geschlechts befindet. Die Bevölkerung des Basisjahres wird nun als nach Alter und Geschlecht getrennter Vektor geschrieben (nachfolgend als Bevölkerungsvektor des jeweiligen Jahres bezeichnet). Der Übergang zum Bevölkerungsvektor des Folgejahres wird, begründet durch das Gesetz der großen Zahlen, als sicherer Eintritt der beiden Lebensereignisse für einen Teil der jeweiligen Altersklasse des jeweiligen Geschlechts betrachtet. Dabei bestimmt die Eintrittswahrscheinlichkeit, für welchen Anteil der Gruppe das Ereignis eintritt. Bei den Fertilitätsraten bedeutet der Nichteintritt des Ereignisses für ein Individuum schlicht, dass kein weiteres Kind geboren wird. Bei den Überlebenswahrscheinlichkeiten hingegen folgt aus dem Nichteintritt des Ereignisses für ein Individuum, dass es verstirbt.

Neben diesen beiden Hauptkomponenten der demografischen Entwicklung gibt es noch einen dritten bestimmenden Faktor für den Übergang ins Folgejahr. Hierbei handelt es sich um die Migration, d.h. um die Außenwanderungen aus und nach Deutschland. Diese beiden Ströme, insbesondere die Immigration, können jedoch nicht durch Eintrittswahrscheinlichkeiten bezogen auf den Stand der inländischen Bevölkerung modelliert werden. Deshalb werden die Anzahl und das Altersprofil der Immigranten und Emigranten eines Simulationsjahres separat mittels einer

2 In diesem Beitrag wird als Basisjahr das Jahr 2010 verwendet. Die Startwerte für die Bevölkerung sind der Bevölkerungsfortschreibung des Statistischen Bundesamtes entnommen. Die Altersklassen werden im gesamten Modell nach einzelnen Altersjahren eingeteilt, bei den Geschlechtern wird zwischen Männern und Frauen unterschieden. 
Szenarioanalyse bestimmt und anschließend zur inländischen Bevölkerung hinzuaddiert bzw. von dieser subtrahiert.

\subsubsection{Lebenserwartung}

Bei den Überlebenswahrscheinlichkeiten ist die Annahme nicht haltbar, dass sie über den gesamten Simulationszeitraum in jeder Altersklasse konstant bleiben. Der in der Vergangenheit beobachtete Anstieg der allgemeinen Lebenserwartung in Deutschland muss auch bei einer Projektion in die Zukunft berücksichtigt werden. Es ist allerdings offen, wie stark sich dieser Vorgang fortsetzen wird. Die Qualität einer Fortschreibungsmethode kann dabei nur daran gemessen werden, wie gut sie in der Vergangenheit dazu in der Lage war, aus noch länger zurückliegenden Daten die jüngere Vergangenheit zu prognostizieren. Ob sich daraus auch schließen lässt, dass dies am aktuellen Rand nach wie vor der Fall ist, ist offen. Dies ist allerdings für jede Prognosetechnik inhärent. Als Fortschreibungsmethode für die Überlebenswahrscheinlichkeiten verwendet dieses Simulationsmodell die Methodik von Bomsdorf \& Trimborn (1992). Sie ist eine einfachere Variante der Methode von Lee \& Carter (1992), die weitgehend dem aktuellen Stand der Forschung entspricht. Durch die Vereinfachung entsteht allerdings zumindest für deutsche Daten kein wesentlicher Verlust an Genauigkeit (Babel et al. 2008: S. 9). Die Methode basiert auf einer Regression des Logarithmus der Sterbewahrscheinlichkeit (also des Gegenstücks zur Überlebenswahrscheinlichkeit) zur Zeitachse:

$$
\ln q_{x t}=a_{x}+b_{x} \cdot t
$$

Hierbei bezeichnet $\mathrm{q}$ die Sterbewahrscheinlichkeit, $\mathrm{x}$ die Altersklasse, $\mathrm{a}$ und $\mathrm{b}$ die beiden Regressionskoeffizienten und t das jeweilige Jahr. Als Daten werden Periodensterbetafeln des Statistischen Bundesamtes der Jahre 1871 bis 2010 verwendet. Aus diesen werden zunächst die Regressionskoeffizienten gewonnen, um die Sterbewahrscheinlichkeiten für jedes Simulationsjahr weiter fortzuschreiben. Anschließend werden letztere in Überlebenswahrscheinlichkeiten umgerechnet und diese für den Übergang ins Folgejahr verwendet.

\subsubsection{Fertilität}

Die Fertilitäten werden aus der Geburtenstatistik des Statistischen Bundesamtes für 2010 (Fachserie 1 Reihe 1.1) entnommen und anschließend über den Simulationszeitraum konstant gehalten. Diese Annahme entspricht zwar nicht vollständig der zu erwartenden Realität, jedoch ist aus den Daten der Vergangenheit kein klarer Trend abzuleiten, und die Veränderungen waren in der jüngsten Vergangenheit auch so gering, dass eine Prognose der Veränderung keinen mit vertretbarem Aufwand 
erreichbaren Qualitätszuwachs für das Modell bedeuten würde. Stattdessen wird mit alternativen Szenarien eine „Was-wäre-wenn-Analyse“ durchgeführt. Des Weiteren wird vereinfachend davon ausgegangen, dass sich die zuwandernde Bevölkerung ab dem Moment, an dem sie zugewandert ist, in ihrem Fertilitätsverhalten nicht mehr von der inländischen Bevölkerung unterscheidet. Dies entspricht der Vorgehensweise bei Holthausen et al. (2012) sowie Werding \& Hofmann (2008).

\subsubsection{Migration}

Für die Modellierung der Migration wird ein komplexerer Ansatz verwendet, als dies in vergleichbaren Simulationsmodellen der Fall ist. Bei diesen wird meist die relative Altersstruktur der Immigranten bzw. der Emigranten konstant gehalten und lediglich ein Pfad für deren jeweilige Gesamtsumme vorgegeben (Werding, 2013: 25-27; Statistisches Bundesamt, 2009: 31-35). ${ }^{3}$ Betrachtet man dagegen die Migrationsströme aus und nach Deutschland in der Vergangenheit, so zeigt sich, dass sich die relative Altersstruktur einerseits teilweise parallel zur Gesamtsumme der Immigranten bzw. Emigranten verändert, und andererseits teilweise eigenen Trends folgt. Deswegen wird eine Parametrisierung der absoluten Altersstruktur mittels einer Gompertz-Funktion verwendet (Vogt \& Althammer, 2015: 42-44). Sie wird für Zuzüge und Fortzüge und dabei jeweils Männer und Frauen getrennt angewandt. Eine Gompertz-Funktion besitzt drei Parameter, von denen einer die Größe der Fläche unter der Kurve (und damit die Summe der Migranten dieses Altersprofils) bestimmt (Parameter A), während der zweite Parameter die Position des Hochpunkts der Kurve verändert (Parameter b), und der dritte Parameter die Linkssteilheit des Profils justiert (Parameter c). Die zu schätzende Zielfunktion lautet dabei folgendermaßen, wobei x die Altersklasse und y die Anzahl der Migranten der Altersklasse bezeichnet ${ }^{4}$ :

$$
y(x)=A \cdot(-b) \cdot \ln (c) \cdot e^{\ln (c) \cdot x-b \cdot c^{x}}
$$

Als Datenbasis dienen die Altersprofile der Migrationsströme aus den Wanderungsstatistiken des Statistischen Bundesamtes von 1991-2011. Mit diesem Datenmaterial werden für jedes dieser Jahre für die vier Migrationsströme die drei Parameter der Gompertz-Funktion mit einer nichtlinearen Kleinste-Quadrate-Methode geschätzt.

3 Dieser Pfad wird außerdem üblicherweise mit einem Wert vorgegeben, der, nach einer kurzen Phase der Anpassung vom Wert im Startjahr auf diesen Wert, dauerhaft gehalten wird.

4 Eine ähnliche Parametrisierung findet sich auch bei Rogers \& Castro (1981), allerdings nicht als Mittel, um Migrationsentwicklungen in die Zukunft fortzuschreiben, sondern zur Analyse von Daten der Vergangenheit. 
Sie werden dann getrennt mit Hilfe von ARMA-Zeitreihen fortgeschrieben. Dabei werden unterschiedliche Szenarien gebildet, die im folgenden Abschnitt erläutert werden. Anschließend wird noch der Abstand zwischen der jeweiligen geglätteten Kurve und dem tatsächlichen Altersprofil der historischen Daten für jedes Jahr von 1991-2011 in Abhängigkeit von der geglätteten Kurve ermittelt. Da dieser über die Zeit hinweg nahezu konstant bleibt, kann sein Durchschnittswert dazu verwendet werden, aus den parametrischen Kurven der zukünftigen Migrationsströme wieder Altersprofile mit ihren typischen Abweichungen von der glatten Kurve herzustellen.

Als Szenarien für die Migrationsströme ergeben sich mehrere Möglichkeiten. Zum einen kann, auch um die Vergleichbarkeit von Ergebnissen zu ähnlichen Simulationsmodellen herzustellen, ein Referenz-Szenario konstruiert werden, in dem der erste Parameter mit einem ARMA (1,2)-Prozess fortgeschrieben wird, während die anderen beiden Parameter konstant auf den Durchschnittswert der Vergangenheit festgesetzt werden. Es gibt aber auch die Möglichkeit, Migrationsschübe oder langsam voranschreitende Veränderungen der Altersstruktur zu modellieren (hierzu sowie für die gesamte Methodik der Parametrisierung der Migration mittels Gompertz-Funktionen Vogt \& Althammer, 2015).

Aus der Addition der über den Leslie-Matrizen-Ansatz projizierten Bevölkerung eines Simulationsjahres und den bis dorthin fortgeschriebenen Zuzügen abzüglich der Fortzüge in jeder Altersklasse ergibt sich dann die Bevölkerung des Folgejahres, so dass sich durch Wiederholung dieses Vorgangs die Bevölkerung bis ins Zieljahr fortschreiben lässt.

\section{2 Ökonomische Simulationsrechnungen zu Erwerbspartizipation und Wirtschaftswachstum}

\subsubsection{Erwerbspartizipation}

Im nächsten Schritt muss ermittelt werden, welcher Anteil jeder Altersgruppe und jeden Geschlechts in den Simulationsjahren dem Arbeitsmarkt als Erwerbspersonen zur Verfügung stehen. Dafür wird eine Vorgehensweise analog zu Burniaux et al. (2004) bzw. zur EU-Kommission (2006) verwendet. Als Datengrundlage dient dabei die Erwerbspartizipationsstatistik des Mikrozensus. Aus dieser werden Erwerbsquoten getrennt nach Alter und Geschlecht gebildet. In einem unechten Kohortenansatz werden aus den Erwerbsquoten anschließend für jedes Jahr Eintrittsraten in den Arbeitsmarkt bzw. Austrittsraten aus dem Arbeitsmarkt gebildet. Diese stellen eine Übergangsrate dar, die beschreibt, wie sich aus dem Anteil der Erwerbspersonen an der Gesamtbevölkerung in einer Alters-/Geschlechtsklasse in einem Jahr der Anteil der Erwerbspersonen an der Gesamtbevölkerung in der 
nächstälteren Alters-/Geschlechtsklasse des Folgejahres ergibt. Von einem unechten Kohortenansatz spricht man deswegen, weil die Raten nicht innerhalb einer Kohorte zwischen zwei Jahren bestimmt werden, sondern innerhalb eines Jahres zwischen zwei benachbarten Altersklassen. Der Fehler ist vernachlässigbar, solange sich die beiden benachbarten Kohorten nicht völlig unterschiedlich verhalten. Von den Eintritts- und Austrittsraten wird nun in jeder Altersklasse der Durchschnitt gebildet. Mit den durchschnittlichen Eintritts- bzw. Austrittsraten in den/aus dem Arbeitsmarkt werden dann aus den Erwerbsquoten jedes Simulationsjahres die Erwerbsquoten des Folgejahres berechnet. Unterhalb einer Altersgrenze geschieht dies mit Hilfe der Eintrittsraten, und oberhalb dieser Grenze mit den Austrittsraten. Die Grenze selbst bestimmt sich als die Altersklasse, oberhalb derer die Eintrittsraten überall null sind. Durch Anwendung der Erwerbsquoten auf die prognostizierte Bevölkerung ergibt sich dann das Erwerbspersonenpotential eines jeden Prognosejahres.

Dieses Verfahren hat den Vorteil, dass es Trends der Erwerbsbeteiligung aus der Vergangenheit fortschreibt, dafür aber den Nachteil, dass die Vorgabe von Annahmen bezüglich eines Abweichens von diesem Trend recht schwer umzusetzen ist. Dies ist allerdings an dieser Stelle unproblematisch, da die einzige vorzugebende Annahme, eine Reaktion auf die Erhöhung des gesetzlichen Renteneintrittsalters sowie auf die vorübergehende Senkung des Eintrittsalters für besonders langjährig Versicherte in diesem Beitrag noch außen vor gelassen wird, so dass noch mit einem gesetzlichen Renteneintrittsalter von 65 Jahren gerechnet wird.

Für die Arbeitslosenquote wird exogen ein Verlauf vorgegeben, der für alle Altersgruppen und beide Geschlechter homogen gilt. Er besitzt denselben konstanten Wert wie bei Werding (2011) von $5 \%$ ab dem Jahr 2020. Vom realen Wert des Jahres 2010 (7,7\%) sinkt die Arbeitslosenquote linear bis 2020 auf diesen Wert ab.

\subsubsection{Makroökonomisches Wachstumsszenario}

Der nächste Schritt zur Projektion der Budgets der gesetzlichen Rentenversicherung ist eine Modellierung der gesamtwirtschaftlichen Entwicklung. In diesem makroökonomischen Wachstumsszenario werden beispielsweise das Wachstum der Arbeitsproduktivität und damit das Bruttolohnwachstum sowie das Wachstum des Bruttoinlandsproduktes für die weitere Simulation ermittelt. Das Vorgehen folgt dabei Werding \& Hofmann (2008). Die Grundlage ist ein Standardmodell der neoklassischen Wachstumstheorie, ein Solow-Swan-Wachstumsmodell. ${ }^{5}$ Dieses beruht auf der Annahme, dass der (exogen gegebene) technische Fortschritt neben der Verfügbarkeit der Produktionsfaktoren Arbeit und Kapital wesentlich das

5 Eine grundlegende Darstellung hierzu findet sich beispielsweise bei Barro \& Grilli (1996). 
Wirtschaftswachstum beeinflusst. Die Produktion wird hier durch einen üblichen Standard-Funktionstyp, eine Cobb-Douglas-Produktionsfunktion beschrieben:

$$
Y_{t}=A_{t} \cdot K_{t}^{\alpha} \cdot\left(h_{t} \cdot L_{t}\right)^{1-\alpha}
$$

Hierbei bezeichnet $\mathrm{Y}$ das jährliche Bruttoinlandsprodukt, A den Stand der technologischen Entwicklung bzw. die totale Faktorproduktivität (TFP). K bezeichnet den zur Verfügung stehenden Kapitalstock, L die Zahl der Erwerbstätigen, und $h$ ein Maß für deren Effektivität bzw. die Auswirkungen ihres durchschnittlichen Qualifikationsgrades auf diese. Und alpha und 1-alpha schließlich bezeichnen die partiellen Produktionselastizitäten der Faktoren Kapital und Arbeit. Über die Abschreibungsquote $\delta$ und die Investitionsquote/Sparquote s wird der Kapitalstock fortgeschrieben:

$$
K_{t}=(1-\delta) \cdot K_{t-1}+s \cdot Y_{t-1}
$$

Entscheidend ist dabei, dass neben dem technischen Fortschritt als Wachstumsursache auch ein Faktor zur Qualität des Humankapitals mit eingerechnet wird. Für diese beiden Wachstumsfaktoren werden konstante Wachstumsraten angenommen, die beide aus der ifo-Produktivitätsdatenbank stammen und letztlich aus Werding \& Hofmann (2008) entnommen wurden. Für die Abschreibungsquote, die Investitions-/Sparquote und die Produktionselastizität des Kapitals werden ebenfalls aus Werding \& Hofmann (2008) Werte entnommen, die dauerhaft konstant gehalten werden. Die Startwerte des Ausgangsjahres für Kapitalintensität, Bruttowertschöpfung und das Bruttoinlandsprodukt sind dagegen der volkswirtschaftlichen Gesamtrechnung des Statistischen Bundesamtes entnommen.

Der unmittelbare Einfluss der Bevölkerungsentwicklung auf das makroökonomische Wachstumsszenario ergibt sich also ausschließlich aus der Anzahl der Erwerbstätigen, die für jedes Simulationsjahr aus dem Erwerbspartizipationsmodul übernommen wird.

\subsection{Modellrechnungen zur gesetzlichen Rentenversicherung}

\subsubsection{Berechnung der Versicherten- und Rentnerzahlen}

Der eigentliche Hauptteil des Simulationsmodells besteht in der Modellierung der Einnahmen- und Ausgabenseite der Budgets der gesetzlichen Rentenversicherung, um so die finanzielle Nachhaltigkeit der gegenwärtigen rentenrechtlichen Regelungen vor dem Hintergrund des demografischen Wandels bestimmen zu können. 
Allerdings sind dabei auch die durchschnittliche Rentenhöhe bzw. der aktuelle Rentenwert und der Rentenbeitragssatz zu ermittelnde Größen, um auch Aussagen über die Tragbarkeit der Entwicklung für den durchschnittlichen Beitragszahler oder Rentenempfänger treffen zu können. Der wesentliche Teil dieses Moduls ist es, für jede das Simulationsmodell durchlaufende Kohorte die durchschnittliche Entgeltpunktezahl zum Renteneintritt zu ermitteln, wobei zu berücksichtigen ist, dass es für die einzelnen Kohortenmitglieder unterschiedliche Renteneintrittszeitpunkte sowie auch unterschiedliche Renteneintrittsanlässe gibt. Für diese Zielvorgaben bietet sich eine Vorgehensweise in Anlehnung an Holthausen et al. (2012) an.

Als erster Schritt sind hierbei zunächst die Zahlen der Erwerbstätigen weiter zu untergliedern, da sie nicht alle in gleichem Maße auch zu den Beitragszahlern gehören. Sie werden zunächst in die Untergruppen der Selbstständigen, der unbezahlt im Haushalt Mithelfenden, der Beamten, der Angestellten und der Arbeiter unterteilt. Ihre Anteile an den Erwerbstätigen des jeweiligen Alters und Geschlechts werden für die Vergangenheit dem Mikrozensus (Fachserie 1 Reihe 4.1.1) entnommen, und der Durchschnitt dieser Quoten über den Zeitraum von 1991-2011 für die Zukunft als konstante Anteile angenommen. Anschließend werden für diese Gruppen dann die Anteile der Versicherten in den Kategorien Selbstständige kraft Gesetz, Selbstständige auf Antrag, versicherungspflichtig Beschäftigte, Empfänger von Arbeitslosengeld, geringfügig Beschäftigte mit Optierung für eine Versicherung sowie freiwillig Versicherte an diesen Erwerbstätigengruppen aus derselben Zeitspanne der Vergangenheit gemittelt und dieser Durchschnitt dann ebenfalls konstant für die Zukunft verwendet. Die Daten für diese Versichertengruppen stammen aus der Versichertenstatistik der Deutschen Rentenversicherung.

Als zweiter Schritt muss für jedes Simulationsjahr die Anzahl der Rentner berechnet werden. Dazu ist es zunächst erforderlich, die Quoten der Versicherten im Verhältnis zur Gesamtbevölkerung in jeder Altersklasse zu berechnen. Anschließend werden daraus die Rentnerquoten errechnet. Unterhalb des Renteneintrittsalters werden sie aus dem Rückgang der Versichertenquoten ab dem Alter von 50 Jahren abgeleitet. Ab dem Renteneintrittsalter werden sie aus der Differenz zwischen 1 und den Versichertenquoten die Rentnerquoten gebildet. Aus diesen und den Bevölkerungszahlen ergibt sich die Anzahl der Rentner jeder Altersklasse.

\subsubsection{Berechnungen zu den akkumulierten Entgeltpunkten}

Nun werden für jede Kohorte die durchschnittlichen persönlichen Entgeltpunkte (mit durchschnittlichem Rentenzugangsfaktor) ermittelt, die ihre Mitglieder bis zum jeweiligen Renteneintritt akkumulieren. Dies wird in vier Schritten durchgeführt. Zunächst muss jedoch vorbereitend noch das (relative) Altersprofil der versicherungspflichtigen Arbeitsentgelte ermittelt werden. Dieses wird aus der 
Statistik der Deutschen Rentenversicherung Bund (Tabelle 76.00 V) ermittelt und über den Zeitraum von 2001-2011 gemittelt. Für den Simulationszeitraum kann dieses Profil als konstant angenommen werden, da die Abweichungen in den Daten der Vergangenheit sehr gering ausfallen. Ebenfalls zur Vorbereitung muss dann anschließend der durchschnittliche Bruttolohn sowie das versicherungspflichtige Durchschnittsentgelt anhand des im makroökonomischen Wachstumsszenario ermittelten Wachstums der Arbeitsproduktivität fortgeschrieben werden.

Im ersten Schritt zur Ermittlung der durchschnittlichen persönlichen Entgeltpunkte zum Renteneintritt jeder Kohorte wird zunächst für jede Kohorte (k) vom Geburtsjahr 1910 bis 2050 protokolliert, wie viele Entgeltpunkte ein durchschnittliches Kohortenmitglied in jedem Simulationsjahr akkumuliert $\left(E P_{1}\right)$. Dies geschieht zunächst getrennt für Erwerbstätige und Bezieher von Arbeitslosengeld. Dazu wird das Verhältnis von im Jahr a versichert beschäftigten Kohortenmitgliedern (vB) zu den versicherten Kohortenmitgliedern (V) mit dem relativen durchschnittlichen Entgelt (rDE) multipliziert, das in der Altersklasse x erreicht wird:

$$
E P_{1}(k, a)=r D E_{x=a-k} \cdot \frac{v B_{k, a}}{\max _{b=k \ldots a} V_{k, b}}
$$

Abweichend von Holthausen et al. (2012) wird dabei jedoch nicht durch die Anzahl der in diesem Jahr versicherten Kohortenmitgliedern geteilt, sondern durch das Maximum der in einem Jahr versicherten Mitglieder dieser Kohorte. Dies hat den Grund, dass ansonsten für ein durchschnittliches Kohortenmitglied angenommen würde, dass es vom Alter 15 bis zum Renteneintritt durchgängig Beiträge zahlen würde. Das ist allerdings unrealistisch, weshalb durch die veränderte Berechnung auch Unterbrechungen oder ein späterer Beginn des Erwerbslebens in die Durchschnittsbildung der Versichertenbiografien mit eingerechnet werden. Ebenfalls wird dadurch in den Durchschnitt mit eingerechnet, dass manche Kohortenmitglieder bereits früher in Rente gehen, und somit nur noch ein kleinerer Teil der Kohortenmitglieder weitere Entgeltpunkte erwirbt. Dies ist für den dritten Schritt der Berechnung relevant, da der Effekt des früheren Renteneintritts mancher Kohortenmitglieder auf das weitere Wachstum der Entgeltpunkte eines durchschnittlichen Kohortenmitglieds dort ansonsten separat berechnet werden müsste. Für die Bezieher von Arbeitslosengeld wird abweichend nicht der Wert aus dem Altersprofil des Durchschnittsentgelts für ihre Altersklasse verwendet, sondern das 0,8-fache des Werts der um ein Jahr jüngeren Altersklasse (damit wird davon ausgegangen, dass die Dauer des Bezugs durchschnittlich ein Jahr dauert). Ansonsten entspricht die Berechnungsmethodik der bei den versicherten Erwerbstätigen.

Als zweiter Schritt findet eine Aufsummierung der Entgeltpunkte jeder Kohorte von Jahr zu Jahr statt, wobei vom Geburtsjahr an in jedem Jahr die durchschnittlich 
neu hinzugewonnenen Entgeltpunkte zu den bis zum Vorjahr insgesamt erreichten Entgeltpunkten addiert werden:

$$
E P_{2}(k, a)=\sum_{b=k}^{a} E P_{1, \text { erwerb }}(k, b)+E P_{1, \text { arbeitslos }}(k, b)
$$

Dabei werden auch die durchschnittlich bei Erwerbstätigkeit und beim Bezug von Arbeitslosengeld erreichten Entgeltpunkte addiert. Außerdem werden in diesem zweiten Schritt Entgeltpunkte, die bereits vor dem Startjahr des Modells erworben wurden, hinzugefügt und ebenfalls mit aufsummiert. Dabei werden ostdeutsche Entgeltpunkte, die aufgrund des unterschiedlichen Berechnungsverfahrens in Westund Ostdeutschland einen anderen Wert haben, in westdeutsche Entgeltpunkte umgerechnet und anschließend in den Kohorten ein Durchschnitt zwischen westund ostdeutschen erworbenen Entgeltpunkten gebildet. Schließlich müssen noch Entgeltpunkte für Kindererziehungszeiten berücksichtigt werden. Für diese wird vereinfachend angenommen, dass sie grundsätzlich von der Mutter in Anspruch genommen werden. Somit kann die Anzahl an Kindern, die von einer Kohorte pro Frau geboren werden, direkt mit der sich durchschnittlich pro Kind ergebenden Entgeltpunktezahl multipliziert werden, und anschließend zur Entgeltpunktesumme bei den Frauen addiert werden.

Der dritte Schritt beinhaltet zunächst eine Ermittlung, wie viele Menschen in welcher Kohorte in welchem Jahr und damit in welchem Alter in die Rente eintreten. Daraus wird berechnet, welchen durchschnittlichen Zugangsfaktor die Rentner jeden Alters in jeder Kohorte haben. Ab dem gesetzlichen Regelrenteneintrittsalter bleibt dieser innerhalb einer Kohorte gleich. Anschließend wird für die Erwerbsminderungsrentner die Entgeltpunktezahl nach dem im SGB VI beschriebenen Verfahren angehoben. Ansonsten werden die Entgeltpunktesummen der jeweiligen Altersklasse direkt mit dem in dieser Kohorte und dieser Altersklasse gültigen durchschnittlichen Zugangsfaktor multipliziert, woraus sich die für die Rentenhöhe maßgeblichen Entgeltpunkte ergeben. Dies gilt allerdings nur für diejenigen Kohorten, deren Mitglieder im Startjahr noch keine Rentner sind. Für die übrigen Kohorten wird aus dem durchschnittlichen Zahlbetrag nach Altersklassen aus der Rentenbestandsstatistik der Deutschen Rentenversicherung für das Startjahr 2010 der für die Rentenzahlung maßgebliche Entgeltpunktestand ermittelt und in die Entgeltpunktestatistik des Modells integriert. Dies geschieht getrennt nach Altersrenten und Erwerbsminderungsrenten.

\subsubsection{Budgetberechungen}

Die Berechnung des Budgets der Rentenversicherung geschieht für jedes Simulationsjahr in zwei Arbeitsschritten. Zunächst wird der aktuelle Rentenwert auf den 
Wert des Folgejahres umgerechnet. Dies geschieht direkt nach den Regelungen des $\$ 68$ SGB VI. Dabei wird auch die sogenannte „Rentengarantie“ berücksichtigt, sofern der aktuelle Rentenwert nach der eigentlichen Anpassungsformel sinken müsste. Im zweiten Schritt werden alle Einnahmen- und Ausgabenbereiche des Budgets berechnet. Für die Alters- und Erwerbsminderungsrenten kann direkt die Summe der mit den jeweiligen Fallzahlen multiplizierten durchschnittlichen Entgeltpunktesummen beim Renteneintritt mit dem Durchschnitt aus aktuellem und vorjährigem aktuellem Rentenwert multipliziert werden. Die zunächst getrennt nach Männern und Frauen berechneten Werte werden dabei addiert. Die Ausgaben für Leistungen zur Teilhabe werden analog zu Holthausen et al. (2012) mit dem Wachstum der Durchschnittsbruttolöhne fortgeschrieben. Die anteiligen Beiträge zur Krankenversicherung der Rentner werden mit dem maßgeblichen Beitragssatz aus den Rentenzahlungen für Alters- und Erwerbsminderungsrenten ermittelt. Die Ausgaben für Hinterbliebenenrenten werden analog zu Holthausen et al. (2012) mit einer festen Wachstumsrate fortgeschrieben, ebenso die Verfahrens- und Verwaltungskosten. Die Grundgesamtheit an beitragspflichtigem Entgelt der Versicherten wird getrennt nach Empfängern von Arbeitslosengeld und versichert Erwerbstätigen berechnet, wobei die Summe der jeweils Versicherten mit dem aktuellen Durchschnittsentgelt bzw. dem 0,8-fachen des letztjährigen Durchschnittsentgelts multipliziert wird. Der Beitragssatz wird, abweichend von der gesetzlichen Regelung, zum Schluss der Budgetrechnung eines Jahres so bestimmt, dass die Beitragseinnahmen ausreichen, die übrig gebliebenen Kosten zu decken. Dafür muss allerdings vorher noch die Höhe der Einnahmen aus den verschiedenen Bundeszuschüssen ermittelt werden. Diese werden nach den in \$213 SGB VI definierten Methoden fortgeschrieben. Anschließend kann der Rentenbeitragssatz festgelegt werden, der das Budget ausgleicht. Zum Schluss wird noch der für das Folgejahr zur Berechnung des allgemeinen Bundeszuschusses maßgebliche fiktive Beitragssatz nach der Formel des $\$ 213$ (2) SGB VI berechnet.

Für diese Budgetrechnungen sind zur Initialisierung einige Startwerte vonnöten, die von unterschiedlichen Quellen übernommen werden müssen. Der aktuelle Rentenwert für das Jahr 2009, der Beitragssatz der Jahre 2009 und 2010 sowie der Ausgleichsfaktor 2010 werden den entsprechenden Verordnungen entnommen, die Rentenausgaben 2009 und 2010 sowie die Beitragseinnahmen beider Jahre entstammen der Statistik der Deutschen Rentenversicherung Bund, ebenso wie die Ausgaben für Rehabilitationsleistungen, die Ausgaben für die Hinterbliebenenrenten und die Einnahmen aus den drei verschiedenen Bundeszuschüssen. 


\section{Ergebnisse der Simulationsrechnungen}

Zur Kalibrierung des Modells wird getestet, ob dieses bei ähnlichen Annahmen auch zu ähnlichen Ergebnissen kommt wie vergleichbare, als realistisch einzustufende Projektionen. Mit den im vorigen Abschnitt beschriebenen Startwerten ist dies in der hier modellierten Referenzvariante, also mit einer nach einer Anpassungsphase konstant bleibenden Migration, bei den wichtigsten Messgrößen der Fall. Als Maßstab bei der Bevölkerungsprognose wird hierzu die 12. koordinierte Bevölkerungsvorausberechnung des Statistischen Bundesamtes verwendet. Sie besteht aus zahlreichen Varianten mit unterschiedlichen Annahmen bezüglich Lebenserwartung, Fertilität und Migration, wobei die Varianten 1-W2 und 2-W2 mit ihren Annahmen am nächsten beim hier modellierten Referenzszenario liegen. So ist die Fertilität gleich wie bei den beiden Varianten. Der Migrationssaldo liegt durch die Zeitreihenprojektion dagegen zunächst höher und passt sich dann auf einen etwas niedrigeren Wert als in den beiden Varianten an. Die Annahme bezüglich der Lebenserwartung liegt etwa mittig zwischen den beiden Varianten. Abbildung 1 zeigt exemplarisch den Verlauf der Bevölkerungsgröße des modellierten Referenz-Szenarios im Vergleich mit den beiden Annahmenvarianten des Statistischen Bundesamtes. Die Ergebnisse zur Bevölkerungszahl sind, verglichen mit der Variante 1-W2, etwas optimistischer und liegen langfristig (bedingt durch die Lebenserwartung) zwischen den beiden Varianten. Kurzfristig ist die Bevölkerungszahl noch höher als die Variante 2-W2, was am zu Beginn größeren Migrationssaldo liegt. Beim Altenquotienten führt ebenfalls der höhere Migrationssaldo zu Beginn zu einem leicht niedrigeren Verlauf als in den beiden Varianten, langfristig ordnet sich das Referenzszenario aber wegen des Verlaufs der Lebenserwartung dazwischen ein. Dies zeigt Abbildung 2. Ein weiterer Vergleich mit anderen Arbeiten insbesondere zur Kalibrierung der ökonomischen Modellbestandteile zeigt ebenfalls ähnliche Werte. Der Altenquotient und die Erwerbspersonenzahl liegen beispielsweise nicht weit entfernt vom Verlauf bei Werding (2011: 8, 30). Bei der Bevölkerungszahl und der Rentnerzahl sind die Werte nahe bei Holthausen et al. (2012: 28f.). Die Anfangswerte der Rentnerzahl entsprechen zudem recht genau den bereits vorliegenden realen Werten der Rentenbestandsstatistik der Deutschen Rentenversicherung.

Im Folgenden werden nun die Ergebnisse der Simulationsrechnungen zu den Auswirkungen des demografischen Wandels im Referenzszenario erläutert. Die erste relevante Messgröße ist dabei die Bevölkerungsgröße. Abbildung 1 zeigt, dass die Bevölkerung ab dem Jahr 2016 zunächst noch langsam, dann aber immer schneller schrumpft. 


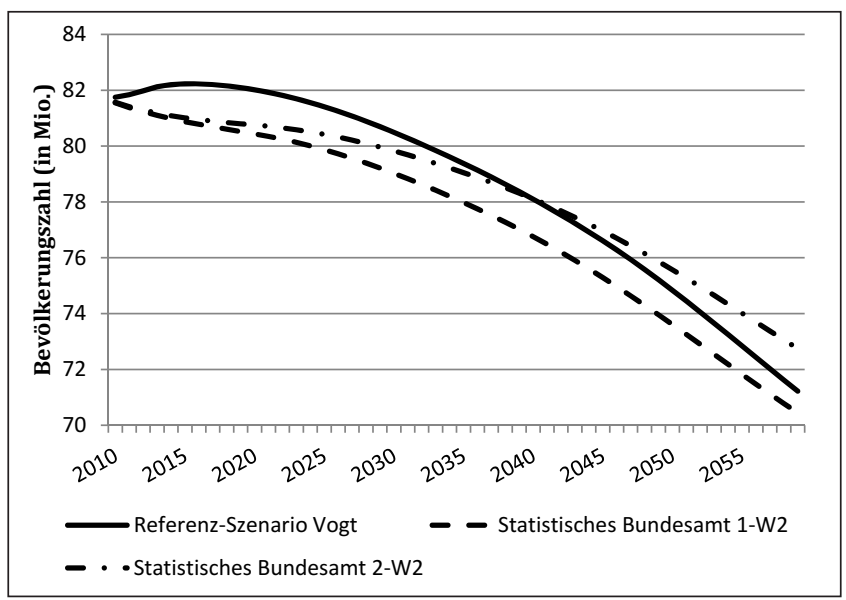

Abb. 1 Bevölkerungszahl (in Mio.)

Quellen: Eigene Berechnung, Statistisches Bundesamt

Der Einfluss dieser Entwicklung auf die gesetzliche Rentenversicherung wird jedoch erst im Zusammenhang mit der Entwicklung des Altenquotienten deutlich (s. Abbildung 2).

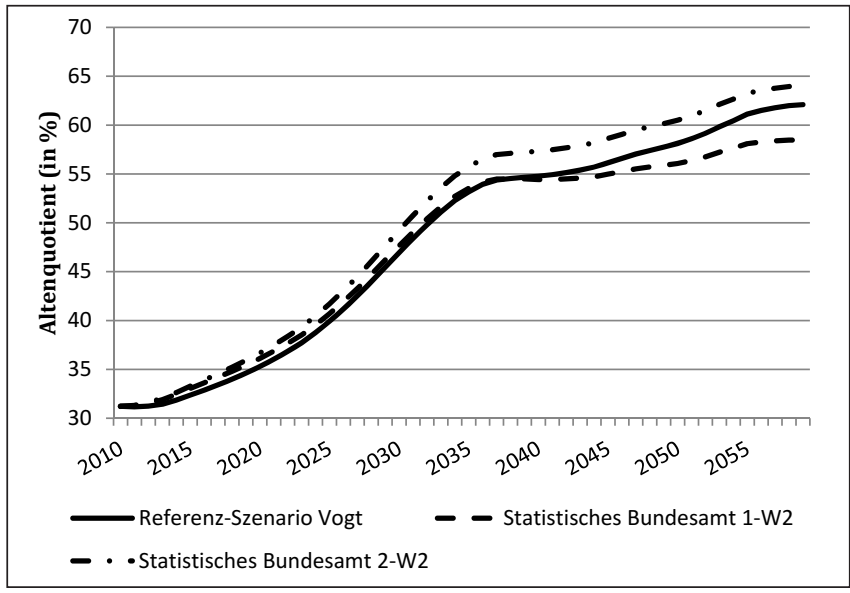

Abb. 2 Altenquotient (in \%)

Quellen: Eigene Berechnung, Statistisches Bundesamt 
Es zeigt sich, dass sich bis etwa 2035 die Alterung der Bevölkerung ebenso beschleunigt wie ihre Schrumpfung. Die geburtenstarken Jahrgänge verlassen in den 2010er Jahren zunehmend den Arbeitsmarkt und werden verrentet, während die deutlich kleineren Kohorten der Geburtsjahrgänge der beiden Weltkriege und der Zwischenkriegszeit langsam versterben. Somit steigt die Anzahl an Menschen im Rentenalter insgesamt an. Die Bevölkerung schrumpft in dieser Zeit noch nicht so stark, da die nachrückenden Neugeborenenjahrgänge zwar eine bereits recht geringe Zahl aufweisen, jedoch auch die Sterbefälle noch eher kleine Jahrgänge betreffen. Die steigende Lebenserwartung führt dazu, dass die geburtenstarken Jahrgänge auch länger Rentner bleiben, als dies bei ihren Vorgängern der Fall war. Auch dadurch beschleunigt sich der Anstieg des Altenquotienten. Die Bevölkerungsabnahme beschleunigt sich dadurch, dass die Anzahl an Frauen im fertilen Alter abnimmt, wodurch die Neugeborenenzahl sinkt. Mit der Zeit versterben dann auch immer geburtenstärkere Jahrgänge, was dazu führt, dass der Anstieg des Altenquotienten in den Jahren von 2035-2040 beinahe zum Erliegen kommt, da keine geburtenstarken Jahrgänge mehr ins Rentenalter nachrücken. Ab 2040 jedoch sinkt die Größe der neu in den Arbeitsmarkt eintretenden Jahrgänge wieder schneller als die Größe der in die Rente eintretenden Jahrgänge, wodurch der Altenquotient wieder stärker ansteigt. Gegen Ende der Simulation deutet sich ein erneutes Abflachen des Anstiegs des Altenquotienten an, während die Bevölkerungsabnahme ungebremst weitergeht.

Die Entwicklung des Altenquotienten zeigt bereits, dass der demografische Wandel massiv die äußeren Rahmenbedingungen für das umlagefinanzierte deutsche Rentensystem verändert. Da in diesem System keine größeren Rücklagen vorgesehen sind und Einnahmen und Ausgaben in jedem Jahr weitgehend übereinstimmen müssen, wirkt sich das verschlechterte Verhältnis zwischen Beitragszahlern und Rentenempfängern direkt auf das Verhältnis zwischen Beitragssatz und aktuellem Rentenwert und damit der durchschnittlichen Rentenhöhe aus. Da in dieser Simulationsrechnung an der Fortschreibungsmethode des aktuellen Rentenwerts und der Bundeszuschüsse nach SGB VI festgehalten wird, schlägt sich dies hauptsächlich im Rentenbeitragssatz nieder, dessen Verlauf eine große Ähnlichkeit zum Verlauf des Altenquotienten aufweist (s. Abbildung 3). 


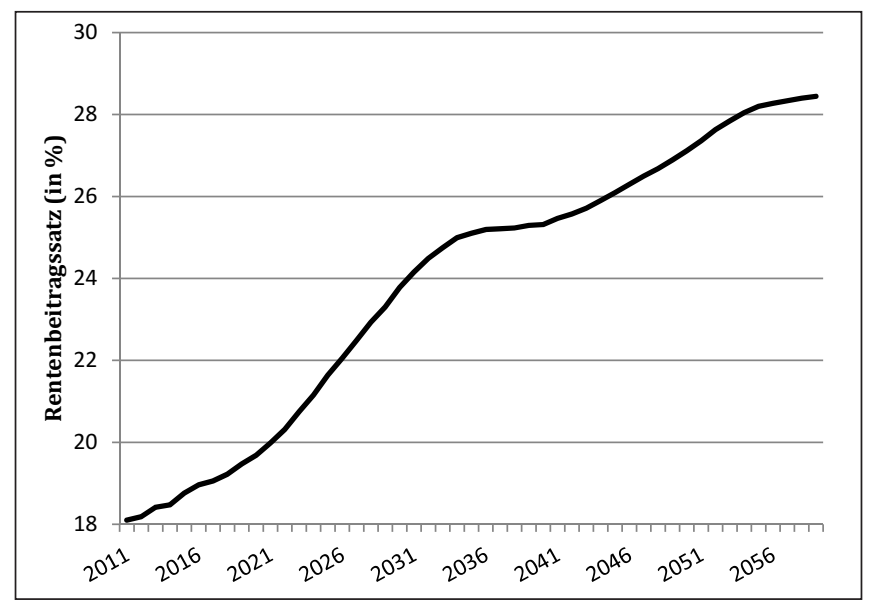

Abb. 3 Rentenbeitragssatz (in \%)

Quelle: Eigene Berechnung

Er zeigt zunächst ebenfalls einen steilen Anstieg bis etwa zum Jahr 2035. Anschließend ergibt sich eine kurze Phase der Stagnation, bevor er ab 2040 wieder steiler ansteigt. Ab 2050 verlangsamt sich der Anstieg dann wieder. Insgesamt steigt er auf Werte von ca. $28 \%$ an, was erheblich über dem bisher für tragbar erachteten Niveau liegt. Das Ziel eines Verbleibs unterhalb der bis 2030 vorgegebenen Grenze von $22 \%$ wird voraussichtlich nicht eingehalten werden können. Ein positives Signal ist jedoch, dass sich nach dem Passieren dieser Grenze der Anstieg bald verlangsamt und die Marke von $25 \%$ wohl erst nach 2040 deutlich überschritten wird. Dementsprechend gibt es bis dahin eventuell die Möglichkeit, Maßnahmen zu ergreifen, um den darauf folgenden steilen Anstieg zumindest etwas abzuflachen. Die Möglichkeiten dafür sind jedoch begrenzt. Eine Änderung des Geburtenverhaltens in einer solch kurzen Zeit ist relativ schwer vorstellbar, jedoch können hier schon sehr kleine Veränderungen große Auswirkungen haben (Holthausen et al., 2012: 34; Althammer, 2014: 101).

Eine andere Möglichkeit der demografischen Beeinflussung wird in einer erweiterten Zuwanderung gesehen. Sie hat aber nur einen überschaubaren Einfluss, sofern sie nicht permanent hohe Werte annimmt, deren Akzeptanz in der Bevölkerung zumindest heute fraglich wäre. Vorstellbar ist es dagegen, dass das Medianalter der Zuwanderung gesenkt werden kann, indem beispielsweise bei einer stärkeren Steuerung der Zuwanderung letztere in höherem Maße auf den Altersbereich am 
Ende der Ausbildung und Beginn der beruflichen Karriere konzentriert werden kann. Eine solche Entwicklung hätte durchaus relevante Auswirkungen auf den Rentenbeitragssatz (Vogt \& Althammer, 2015: 48). Weitere Möglichkeiten einer Anpassung wären eine Veränderung der Berechnung des aktuellen Rentenwerts (was einer weiteren Absenkung des durchschnittlichen Rentenniveaus entspräche), sowie eine weitere Erhöhung des Renteneintrittsalters, die teilweise bereits in der Diskussion ist (Börsch-Supan, 2014: 11). Eine Neuausrichtung der gesetzlichen Rentenversicherung auf eine stärkere Berücksichtigung der Kinderzahl (Sinn, 1997: 8; Werding, 2003: 209) hin könnte dagegen frühestens in der zweiten Hälfte des 21. Jahrhunderts die demografische Situation verändern, sofern sie überhaupt einen nennenswerten Einfluss auf das Geburtenverhalten generiert. Eher ist von ihr zu erwarten, dass sie zu einer erheblichen Rentenniveausenkung für die Jahrgänge führen kann, die zum Zeitpunkt der Reform ihr fertiles Alter bereits verlassen haben.

Eine wichtige Frage ist allerdings auch noch, ob die nach den Vorgaben des SGB VI fortgeschriebenen Bundeszuschüsse so für den Bund dauerhaft tragbar bleiben. Die Fortschreibungsformeln ergeben ein höchst unterschiedliches Ausmaß des Einflusses des demografischen Wandels auf die einzelnen Bundeszuschüsse. Der zusätzliche Bundeszuschuss ist nur an das Wachstum der Durchschnittsbruttolöhne gekoppelt, kann also allenfalls moderat ansteigen. Der Erhöhungsbetrag zum zusätzlichen Bundeszuschuss ist, neben der Entwicklung der Durchschnittsbruttolöhne, auch von der Entwicklung der Anzahl der nicht selbstständig Beschäftigten abhängig, wodurch sein Anstieg durch den demografischen Wandel sogar beschränkt wird. Die „echten Beitragszahlungen“ des Bundes für Kindererziehungszeiten sind in ihrem Wachstum durch die sinkende Kinderzahl ebenfalls vom demografischen Wandel gebremst, gleichzeitig allerdings auch durch die Kopplung an die Beitragssatzerhöhung wieder beschleunigt. Einzig der allgemeine Bundeszuschuss wird durch die Berechnung mit Hilfe des fiktiven Beitragssatzes durch den Verlauf des demografischen Wandels gegenüber der Wachstumsrate der Durchschnittsbruttolöhne in erheblichem Maße zusätzlich erhöht.

Die Berechnungen (s. Abbildung 4) zeigen beim allgemeinen Bundeszuschuss auch den stärksten Anstieg, der auch einen zu Altenquotient und Rentenbeitragssatz ähnlichen Verlauf aufweist, mit einem sich zunächst bis 2030 verstärkenden Anstieg, dann einer Verlangsamung insbesondere von 2035 bis 2042, und schließlich wieder einem stärkeren Anstieg, der zum Ende hin wieder den Beginn einer Abflachung zeigt. Insgesamt verdreifacht er sich bis 2059 in etwa, während sich der zusätzliche Bundeszuschuss und sein Erhöhungsbetrag nur in etwa verdoppeln. Da sich im makroökonomischen Wachstumsszenario das Bruttoinlandsprodukt nicht ansatzweise verdoppelt, sondern nur etwa um den Faktor 1,6 vergrößert, erscheint es wahrscheinlich, dass die Bundeszuschüsse für die gesetzliche Rentenversicherung 


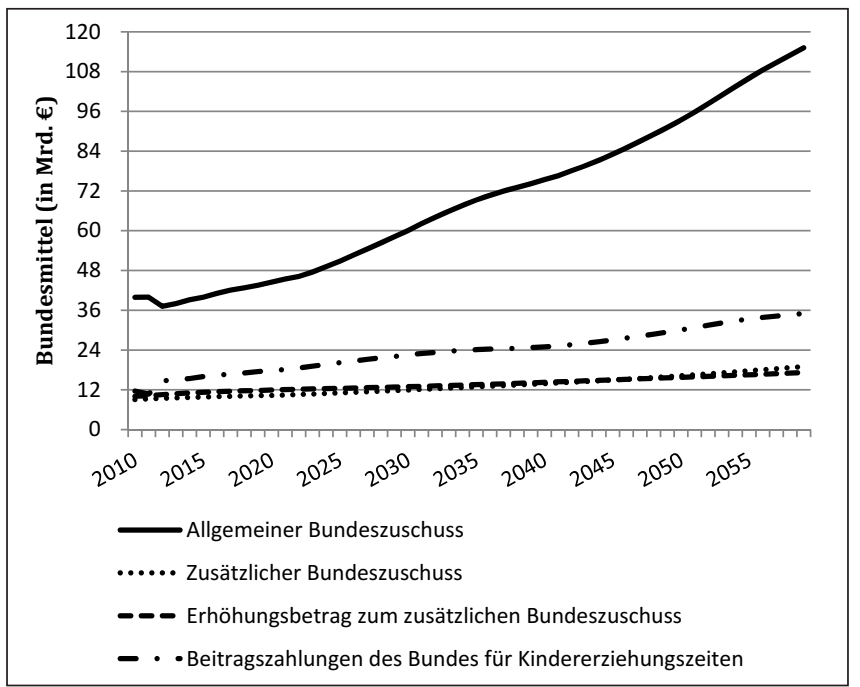

Abb. 4 Bundesmittel zur Finanzierung der GRV (in Mrd. €)

Quelle: Eigene Berechnung

bis dahin einen wesentlich größeren Anteil des Bundeshaushalts ausmachen werden, als dies heute der Fall ist. Da ein recht großer Teil der Steuerzahler auch gleichzeitig Rentenbeitragszahler ist, bedeutet dies also eine noch größere Belastung für die erwerbstätige Bevölkerung als durch die Beitragslast ohnehin schon, da diese Bundesmittel entweder durch zusätzliche Steuereinnahmen finanziert werden müssen oder durch Einsparungen in anderen Bereichen des Bundeshaushalts.

\section{$4 \quad$ Fazit}

Die Simulation der Entwicklung der Beiträge und Bundeszuschüsse zur gesetzlichen Rentenversicherung zeigt, dass die deutschen umlagefinanzierten Sicherungssysteme derzeit nicht nachhaltig ausgestaltet sind. Der prognostizierte Anstieg des Beitragssatzes auf etwa $28 \%$ sowie die deutliche Zunahme des erforderlichen Bundeszuschusses weisen eine massive Überbelastung zukünftiger Generationen aus. Nach den hier vorgelegten Berechnungen übersteigt der erforderliche Beitragssatz zur Rentenversicherung die mittelfristige Zielgröße von 22 \% bereits im Jahr 2026, 
und dies, obwohl in diesem Simulationsmodell mit Ausnahme der Anhebung des Renteneintrittsalters auf 67 Jahre bereits die jüngsten Maßnahmen zur langfristigen Stabilisierung der gesetzlichen Rentenversicherung berücksichtigt sind. Auch die Absenkung des durchschnittlichen Rentenniveaus im Zuge der „Riesterrente " und der Einführung des Nachhaltigkeitsfaktors sind offensichtlich unzureichend, um das langfristige demografische Problem zu lösen. Dies ist umso problematischer, als eine weitere Absenkung des durchschnittlichen Rentenniveaus vermutlich nicht in Frage kommen dürfte. Denn bereits unter den gegebenen Bedingungen ist die beitragsfinanzierte Alterssicherung nur noch unzureichend in der Lage, zukünftig Altersarmut zu vermeiden. Eine weitere Absenkung des durchschnittlichen Rentenniveaus würde somit die gesellschaftliche Akzeptanz der gesetzlichen Rentenversicherung in Frage stellen.

Die langfristige demografische Entwicklung macht eine grundlegende Reform der Rentenversicherung erforderlich. Mit Hilfe des im Rahmen des Forschungsprojekts „Ein dynamisches Mikrosimulationsmodell zur Evaluation der Nachhaltigkeit sozialer Sicherungssysteme" entwickelten Simulationsmodells ist es möglich, unterschiedliche Politiken auf ihren Beitrag zur Stabilisierung der Rentenversicherung $\mathrm{zu}$ untersuchen. In Frage kommen eine weitere Verlängerung der Lebensarbeitszeit, Veränderungen bei der Anrechnung rentenrechtlicher Zeiten oder eine unterschiedliche Gewichtung dieser Versicherungszeiten. Eine Analyse dieser und weiterer Fragestellungen muss jedoch zukünftigen Arbeiten überlassen bleiben.

\section{Literatur}

Auerbach, A.J. \& Kotlikoff, L.J. (1987). Dynamic Fiscal Policy. Cambridge, MA: Cambridge University Press.

Althammer, J. (2014). Nachhaltige Sozialpolitik. Aufgaben der Sozial- und Familienpolitik angesichts der demografischen Entwicklung. In: A. Kruse, G. Maio \& J. Althammer (Hrsg.), Humanität einer alternden Gesellschaft (pp. 81-101). (Veröffentlichungen der Joseph-Höffner-Gesellschaft, Bd. 3). Paderborn: Ferdinand Schöningh.

Babel, B., Bomsdorf, E. \& Schmidt, R. (2008). Forecasting German mortality using panel data procedures. Journal of Population Economics, 21(3): 541-555.

Barro, R.J. \& Grilli, V. (1996). Makroökonomie: Europäische Perspektive. Wolls Lehr- und Handbücher der Wirtschafts- und Sozialwissenschaften. München: Oldenbourg.

Bomsdorf, E. \& Trimborn, M. (1992). Sterbetafel 2000. Modellrechnungen der Sterbetafel. Zeitschrift für die gesamte Versicherungswissenschaft, 81(3): 457-481.

Börsch-Supan, A. (2014). Ökonomie einer alternden Gesellschaft. Perspektiven der Wirtschaftspolitik, 15(1): 4-23. 
Burniaux, J.-M., Duval, R. \& Jaumotte, F. (2004). Copeing with Ageing: A Dynamic Approach to Quantify the Impact of Alternative Policy Options on Future Labour Supply in OECD Countries. OECD Economics Department Working Papers 371.

Caswell, H. (2001). Matrix Population Methods: Construction, Analysis, and Interpretation. Sunderland, MA: Sinauer Associates.

EU-Kommission (2006). The 2005 projections of age-related expenditure (2004-50) for the EU-25 Member States: underlying assumptions and projection methodologies. Special Report 4/2005: European Economy. European Commission Directorate-General for Economic and Financial Affairs.

Fehr, H., Jokisch, S. \& Kotlikoff, L. (2005). The Developed World's Demographic Transition - The Roles of Capital Flows, Immigration, and Policy. In: R. Brooks \& A. Razin (Hrsg.), Social Security Reform - Financial and Political Issues in International Perspective (pp. 11-43). New York: Cambridge University Press.

Holthausen, A., Rausch, J. \& Wilke, C. (2012). MEA-PENSIM 2.0: Weiterentwicklung eines Rentensimulationsmodells, Konzeption und ausgewählte Anwendungen. MEA Discussion Papers 254-2012.

Kommission zur Nachhaltigkeit der sozialen Sicherungssysteme (2003). Nachhaltigkeit in der Finanzierung der sozialen Sicherungssysteme. Bericht der Kommission. Berlin: Bundesministerium für Gesundheit und Soziale Sicherung.

Lee, R. D. \& Carter, L. R. (1992). Modeling and Forecasting U.S. Mortality. Journal of the American Statistical Association, 87(419): 659-671.

Rogers, A. \& Castro, L. J. (1981). Model Migration Schedules. Research Report RR-81-30. Laxenburg: International Institute for Applied Systems Analysis.

Sinn, H. W. (1997). The Value of Children and Immigrants in a Pay-as-you-go Pension System: A Proposal for a Partial Transition to a Funded System. CES Working Paper 141.

Statistisches Bundesamt (2009). Bevölkerung Deutschlands bis 2060. 12. koordinierte Bevölkerungsvorausberechnung. Begleitmaterial zur Pressekonferenz am 18. November 2009 in Berlin.

Vogt, V. \& Althammer, J. (2015). Zuwanderung nach Deutschland: Makroökonomische Szenarioanalyse der Auswirkungen verstärkter Migration auf Altenquotient und Beitragssatz zur gesetzlichen Rentenversicherung. DGD-Onlinepublikation 01/2015: 38-49.

Werding, M. (2003). Rente nach Kinderzahl: Argumente zugunsten einer unpopulären Idee. Zeitschrift für Wirtschaftspolitik, 52: 204-214.

Werding, M. (2011). Demographie und öffentliche Haushalte. Simulationen zur langfristigen Tragfähigkeit der gesamtstaatlichen Finanzpolitik in Deutschland. Arbeitspapier 03/2011. Sachverständigenrat zur Begutachtung der gesamtwirtschaftlichen Entwicklung.

Werding, M. (2013). Modell für flexible Simulationen zu den Effekten des demographischen Wandels für die öffentlichen Finanzen in Deutschland bis 2060: Daten, Annahmen und Methoden. Dokumentation im Auftrag der Bertelsmann-Stiftung. Ruhr-Universität Bochum.

Werding, M. \& Hofmann, H. (2008). Projektionen zur langfristigen Tragfähigkeit der öffentlichen Finanzen. ifo Beiträge zur Wirtschaftsforschung 30. 
Open Access Dieses Kapitel wird unter der Creative Commons Namensnennung - Nicht kommerziell 2.5 International Lizenz (http://creativecommons.org/licenses/by-nc/2.5/deed. de) veröffentlicht, welche für nicht kommerzielle Zwecke die Nutzung, Verbreitung und Wiedergabe in jeglichem Medium und Format erlaubt, sofern Sie den/die ursprünglichen Autor(en), den Titel des Werks und die Quelle ordnungsgemäß nennen, einen Link zur Creative Commons Lizenz beifügen und im Falle einer Abwandlung durch einen entsprechenden Hinweis deutlich erkennbar machen, dass Änderungen vorgenommen wurden.

Die in diesem Kapitel enthaltenen Bilder und sonstiges Drittmaterial unterliegen ebenfalls der genannten Creative Commons Lizenz, sofern sich aus der Abbildungslegende nichts anderes ergibt. Sofern das betreffende Material nicht unter der genannten Creative Commons Lizenz steht und die betreffende Handlung nicht nach gesetzlichen Vorschriften erlaubt ist, ist auch für die oben aufgeführten nicht-kommerziellen Weiterverwendungen des Materials die Einwilligung des jeweiligen Rechteinhabers einzuholen. 